\title{
Tratamento cirúrgico da compressão do esôfago por anomalia do arco aórtico: relato de caso
}

Ivo RICHTER*, Hélio Pereira MAGALHĀES*, Luiz Miguel CORTEZ*, Douglas GONÇALVES*, Marli GONÇALVES*

\section{RBCCV 44205-142}

\begin{abstract}
RICHTER, I.; MAGALHĀES, H. P.; CORTEZ, L. M.; GONÇALVES, D.; GONÇALVES, M. - Tratamento cirúrgico da compressão do esôfago por anomalia do arco aórtico: relato de caso. Rev. Bras. Cir. Cardiovasc., 6(2): 128-131, 1991.

RESUMO: Paciente adulta, portadora de anomalia vascular congênita do arco aórtico com formação de anel envolvendo traquéia e esôfago, com compressāo deste e conseqũente disfagia progressiva. A abordagem cirúrgica foi realizada por toracotomia lateral direita, com ressecção do divertrículo de Kommerell, secção do ligamento arterioso e ligadura simples da artéria subclávia, com regressão total dos sintomas. Nāo foi observada síndrome de roubo da subclávia ou isquemia do membro superior esquerdo. Os autores discutem o tipo de abordagem e cirurgia para tratamento desta rara anomalia.
\end{abstract}

DESCRITORES: arco aórtico, anomalia, cirurgia; esôfago, compressão, cirurgia.

\section{INTRODUÇÃO}

A primeira citação feita sobre anomalia congênita do arco aórtico com formação de anel vascular compressivo, foi relatada por Bayford em 1787, quando da análise de uma necropsia de mulher com 62 anos de idade, que havia falecido em extrema caquexia devido a importante obstrução do esôfago por artéria subclávia direita anômala. Este fato foi denominado de disfagia lusória.

Vários tipos de anéis vasculares são descritos na literatura $7,8,{ }^{10}$, sendo mais comuns: o duplo arco aórtico direito e/ou esquerdo; 0 arco aórtico à direita com imagem em espelho dos ramos; 0 arco à direita com subclávia esquerda aberrante com ou sem divertículo de Kommerell (Figura 1); e arco aórtico à esquerda com subclávia direita aberrante. Apesar da maioria ter o diagnóstico e o tratamento cirúrgico bem definidos, especial atenção deve ser dada à anomalia do arco aórtico à direita com subclávia esquerda aberrante retroesofágica originária de divertículo de KOMMERELL ${ }^{9}$, com ligamento arterio-

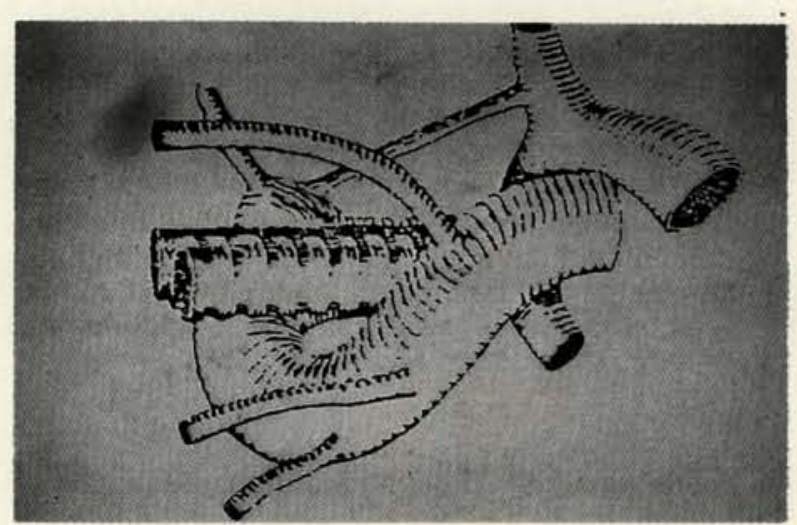

Fig. 1 - Arco aórtico à direita com subclávia esquerda aberrante e diverticulo de Kommerell.

Trabalho realizado no Hospital Santa Paula. Săo Paulo, SP, Brasil.

Recebido para publicaçăo em 14 de maio de 1991.

- Do Hospital Santa Paula.

Endereço para separatas: Ivo Richter. Rua Joăo de Souza Dias, 757, Apto.: 101. 04618 Campo Belo, São Paulo, SP, Brasil. 
RICHTER, I.; MAGALHĀES, H. P.; CORTEZ, L. M.; GONÇALVES, D.; GONÇALVES, M. - Tratamento cirúrgico da compressão do esôfago por anomalia do arco aórtico: relato de caso. Rev. Bras. Cir. Cardiovasc., 6(2): 128-131, 1991.

so ${ }^{10}$. Esta anomalia é rara e sua abordagem cirúrgica e manipulação das estruturas necessitam avaliação criteriosa. Anomalias do arco aórtico com formaçăo de anel vascular compressivo tráqueo-esofágico são, na sua grande maioria, diagnosticados em recém-nascidos $e$ lactentes, sendo seus principais sintomas a tosse, a dispnéia e a regurgitação ${ }^{2}$. Já no adulto, os sintomas são mais relacionados à compressão esofágica, pois a rigidez dos anéis cartilaginosos da traquéia não permite a sua deformação, e sendo assim, a disfagia progressiva com comprometimento do estado geral é a regra.

\section{RELATO DO CASO}

F. M. M. P. T., feminina, 39 anos de idade, cor branca, brasileira, psicóloga, submetida a primeira consulta ambulatorial em $01 / 88$, referindo disfagia progressiva, com piora há três meses. Atualmente só ingere líqüidos e apresenta perda de peso de $6 \mathrm{~kg}$.

Durante a anamnese a paciente relata que desde a infância apresentava dificuldades à deglutição. Nascida de parto gemelar, sendo que a irmã faleceu alguns dias após o parto de "doença azul".

Ao exame físico apresentava-se eupnéica, acianótica, anictérica, mucosas descoradas $+/++$ e palidez cutânea - freqüência cardiaca de 100 bpm; pressão arterial de 110/80. Coração com bulhas normais sem sopros; pulmões limpos sem ruídos adventícios; abdome sem hepatoesplenomegalia. Pulsos presentes e palpáveis bilateralmente (carotídeos, radiais, femorais, popliteos e pediosos).

Os exames complementares revelaram discreta anemia com leucograma normal; a radiografia de tórax $(02 / 88)$ revelava área cardíaca normal com arco aórtico à direita associado a vaso anômalo que condiciona compressão esofágica no $1 / 3$ médio, no sentido pósteroanterior. O exame endoscópico mostrava: esôfago; progressão fácil, trajeto e motilidade conservados. $\mathrm{Na}$ altura da carina, notava-se abaulamento do tipo compressão extrínseca sugerindo alteração vascular. Não há estenose da luz; a laringobroncofibroscopia mostrava hipotonia de cordas vocais. normal.

O ecocardiograma bidimensional com Doppler era

O estudo angiográfico - mostra aorta descendente à direita, valva aórtica trivalvulada e competente, ausência do tronco braquiocefálico, subclávia e carótida direitas emergindo anteriormente e separadas; a carótida esquerda nasce também anteriormente e artéria subclávia esquerda emerge posteriormente no início da aorta descendente em uma região dilatada compativel com a descrição angiográfica do divertículo de Kommerell.

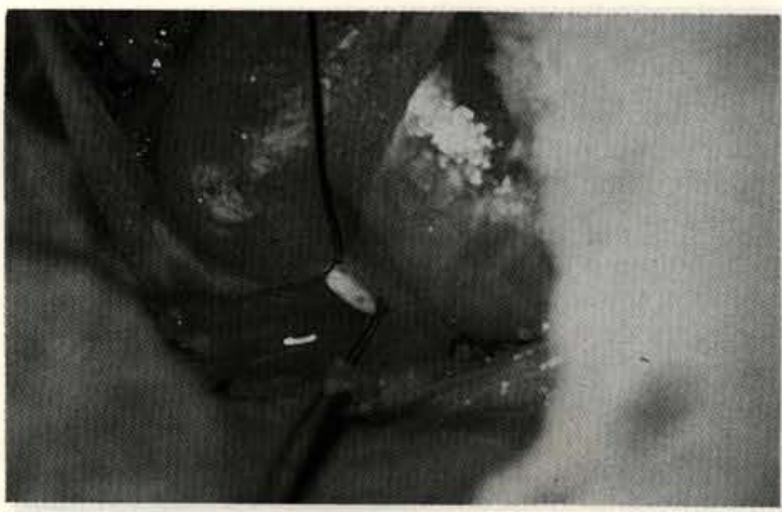

Fig. 2 - Ligadura e posterior secção do ligamento arterioso que emerge da base direita do divertículo de Kommerell.

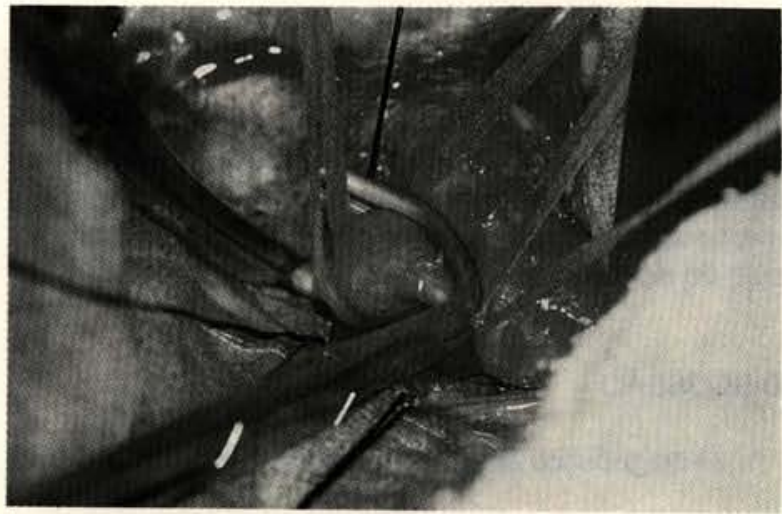

Fig. 3 - Clampeamento do divertículo de Kommerell proximalmente e distalmente ao clampeamento da artéria subclávia esquerda.

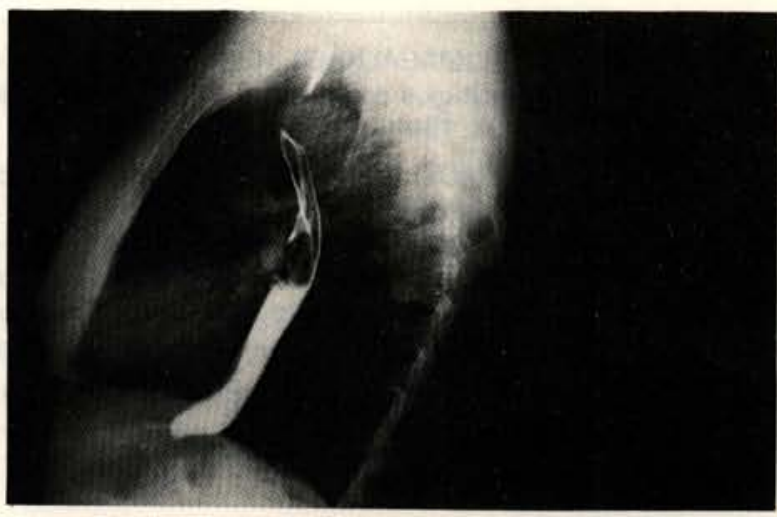

Fig. 4 - Radiografia em PE mostrando compressão importante do esô. fago pelo divertículo e deslocamento anterior de traquéia.

A cirurgia foi realizada através de toracotomia póstero lateral direita no $4^{\circ}$ espaço intercostal, secção da veia ázigos, ligadura e secção do ligamento arterioso (Figura 2), ressecção do divertículo de Kommerell por pinçamento lateral da aorta com pinça de Satinsky (Figura 3) e ligadura simples de artéria subclávia esquerda retroesofágica. 
RICHTER, I.; MAGALHĀES, H. P.; CORTEZ, L. M.; GONÇALVES, D.; GONÇALVES, M. - Tratamento cirúrgico da compressão do esôfago por anomalia do arco aórtico: relato de caso. Rev. Bras. Cir. Cardiovasc., 6(2): 128-131, 1991.

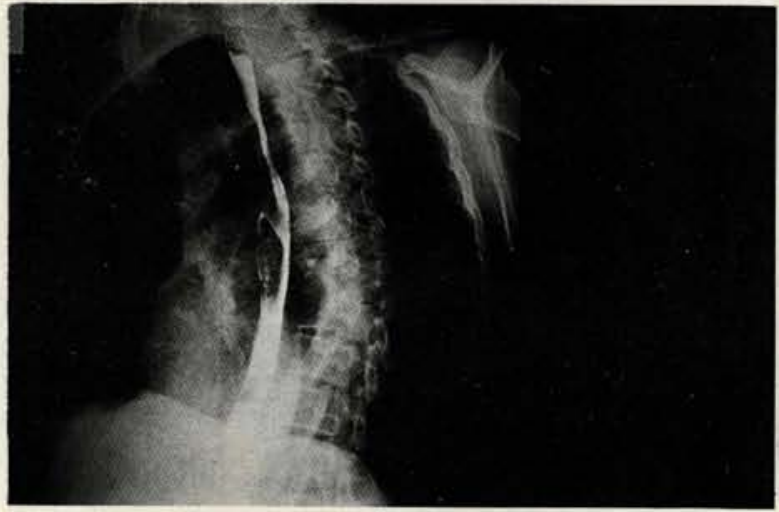

Fig. 5 - Radiografia em OAE mostrando tanto traquéia quanto esôfago livres de deslocamento e da compressāo extrínseca do divertículo.

No pós-operatório imediato, observou-se regressão total da sintomatologia, passando a paciente a ingerir qualquer tipo de alimento.

As figuras 4 e 5 mostram o estudo radiológico comparativo pré e pós-operatório indicando o desaparecimento da compressāo extrínseca do esôfago pelo divertículo de Kommerell.

\section{DISCUSSÃO}

O diagnóstico anatômico preciso das anomalias do arco aórtico é muito importante para a escolha da abordagem cirúrgica.

Ao optarmos pela toracotomia póstero-lateral direita dispúnhamos de todos os elementos que cumprimiam o esôfago, com facilidade de manipulação, pois a compressão, neste caso, fez-se principalmente devido ao divertículo de Kommerell ${ }^{8}$, 11 e ao ligamento arterioso, que unia a base do divertículo à artéria pulmonar esquerda.

A literatura ${ }^{8,11}$ recomenda, nos casos de arco aórtico à direita com subclávia anômala esquerda emergente de divertículo de Kommerell, a toracotomia direita, a secção do ligamento arterioso e a ressecção do divertículo de Kommerell. O reimplante da artéria subclávia esquerda nāo foi realizado no nosso caso porque, ao ser a mesma dispinçada, o fluxo de sangue foi considerado excelente, evitando-se a anastomose, que poderia ser feita na aorta descendente ou subclávia D, com interposição de material protéico. Não foi notada síndrome de roubo ou isquemia do membro superior esquerdo, sendo que no primeiro dia de pós-operatório havia pulso amplo na artéria radial esquerda.

No adulto não há a necessidade da ressecção da artéria subclávia em posição posterior ao esôfago.

A toracotomia esquerda com divisão da artéria subclávia esquerda, secção do ligamento arterioso e restabelecimento do fluxo direto da artéria subclávia esquerda é indicado na ausência de divertículo de Kommerell.

RBCCV 44205-142

RICHTER, I.; MAGALHĀES, H. P.; CORTEZ, L. M.; GONÇALVES, D.; GONÇALVES, M. - Surgical treatment of esophagus compression by aortic arch anomaly: case report. Rev. Bras. Cir. Cardiovasc., 6(2): 128-131, 1991.

ABSTRACT: An adult patient presenting a congenital vascular anomaly of the aortic arch and ring formation, surrounding trachea and esophagus, by compression of the latest was complaining of progressive dysphagia. A surgical approach was performed by right lateral thoracotomy with ressection of the Kommerell diverticulum, section of the arterious ligament and single ligation of the subclavian artery which was followed by total regression of the symptoms. No subclavian steal syndrome or ischemia was observed on the left upper limb. The authors discuss the manner of approach and surgical treatment of this rare anomaly.

DESCRIPTORS: aortic arch anomaly, surgery; esophagus compression, surgery.

\section{REFERÊNCIAS BIBLIOGRÁFICAS}

1 BERENZWEIG, H.; BAUE, A. E.; McCALLUM, R. W. Dysphagia Lusoria: report of a case and review of the diagnostic and surgical approach. Digest. Dis. Sci., 25: $630,636,1980$.

2 BINET, J. P. \& LANGLOSIS, J. - Aortic arch anomalies in children and infants. J. Thorac. Cardiovas. Surg., 73: 248-252, 1977.
3 EDUARDO, A.; MEHDI, H.; JACK, H. H.; ZIA. Q. F.; EDWARD, W. G. - Surgical management of congenital vascular ring. J. Thorac. Cardiovasc. Surg., 77: 721-727, 1979.

4 EDWARDS, J. E. - Anomalies of the derivatives of aortic arch system. Med. Clin. N. Am., 32: 925-947, 1948.

5 GODTFREDSEN, J.; WENNEVOLD, F. E.; LAURIDSEN, P. - Natural history of vascular ring with clinical mani- 
RICHTER, I.; MAGALHĀES, H. P.; CORTEZ, L. M.; GONÇALVES, D.; GONÇALVES, M. - Tratamento cirúrgico da compressão do esôfago por anomalia do arco aórtico: relato de caso. Rev. Bras. Cir. Cardiovasc., 6(2): 128-131, 1991.

festations. Scand. J. Thorac. Cardiovasc. Surg., 11: 75-77, 1977.

6 HALLMAN, G. L. \& COOLEY, D. A. - Congenital vascular ring: surgical considerations. Arch. Surg., 88: 666-675, 1964.

7 HARRY, S. - A new operation for symptomatic aberrant right subclavian artery in the adult. (dysphagia lusoria). J. Thorac. Cardiovasc. Surg., 57:269-272, 1969.

8 JUNG, J. Y.; CARL, H. A.; SALIM, B. S.; LABABIDI, $Z$. - Surgical repair of right aortic arch with aberrant left subclavian artery and left ligamentum arteriosuns. J. Thorac. Cardiovasc. Surg., 75: 237-243, 1978.

9 KOMMERELL, B. - Verlagerung des Oesophagus durch eine abnorm Verlaufende Arteria subclavia dextra (Arteria lusoria). Fortschr. Geb. Rontgenstr., 54: 590-595, 1936.

10 LEE, D. R.; LOUIS, D. L.; WILLIAM, M. K.; MONOUCHER, F. - Aortic arch anamalies in adult disorders of deglutition. Ann. Otol., 87: 498-508, 1978.

11. WONG, P. H. C. \& LEE, J. N. T. - Right aortic arch Kommerell's diverticulum. Thorax, 38: 553-555, 1983. 